\title{
ERGODIC AND SPECTRAL ANALYSIS OF CERTAIN INFINITE MEASURE PRESERVING TRANSFORMATIONS
}

\author{
WILLIAM PARRY
}

0. Introduction. Throughout this paper $T$ will denote a measure preserving transformation on a $\sigma$-finite infinite measure space $(X, B, m)$ which is point isomorphic with the Lebesgue measure space of the real line. Unless otherwise stated, $T$ will be one-one. Equations involving functions or sets will always be interpreted modulo sets of measure zero.

$T$ is said to be ergodic if $T^{-1} E=E, E \in B$, implies either $m E=0$ or $m(X-E)=0$.

P. R. Halmos [1] has posed the problem of characterising spectrally the property of ergodicity for $\sigma$-finite infinite measure preserving transformations. Our purpose is to show that the ergodicity of such a transformation is not a spectral property. Let $U_{T}$ denote the induced unitary operator of $L_{2}(X, B, m)$ onto itself defined by

$$
U_{T}: f(x) \rightarrow f(T x)
$$

for $f(x) \in L_{2}(X, B, m)$. We produce two infinite measure preserving transformations $S, T$ one of which is ergodic and the other not ergodic, such that

$$
V U_{S} V^{-1}=U_{T}
$$

for some unitary operator $V$ of $L_{2}(X, 囚, m)$ on to itself.

Our examples are produced after an analysis of infinite measure preserving transformations which satisfy conditions analogous to Kolmogorov's zero-one law. Extending the current terminology [2], we call these transformations infinite $K$-automorphisms. Unlike the finite $K$-automorphisms, infinite $K$-automorphisms are not necessarily ergodic, for they may possess wandering sets of positive measure. However, if no wandering sets of positive measure exist, in fact if the conservative part of the transformations is not null, then an infinite $K$-automorphism is ergodic. In any case all infinite $K$-automorphisms have denumerable Lebesgue spectrum, and a completely dissipative transformation has denumerable Lebesgue spectrum. (There is no need to speak of the orthogonal complement of the constant functions when the measure space is infinite since nonzero constant func-

Received by the editors June 6, 1964. 
tions are not square integrable.) To prove our main result it suffices to produce an ergodic infinite $K$-automorphism and a completely dissipative transformation. Both transformations have denumerable Lebesgue spectrum and are therefore unitarily equivalent. Concretely, the nonergodic transformation $S$ of the real line defined by

$$
S: x \rightarrow x+1
$$

and the ergodic shift transformation $T$ associated with the random walk on the integers (with equal probabilities of $1 / 3$ moving one step to the left, remaining where it is, or moving one step to the right) are unitarily equivalent.

1. Definitions. Two unitary operators $U_{1}, U_{2}$ of $L_{2}(X, B, m)$ onto itself are said to be unitarily equivalent if there exists a unitary operator $V$ of $L_{2}(X, B, m)$ onto itself such that

$$
V U_{1} V^{-1}=U_{2} \text {. }
$$

A unitary operator $U$ of $L_{2}(X, \beta, m)$ is said to have denumerable Lebesgue spectrum if there exists an orthonormal basis $\left\{f_{i, j}\right\}(i=0,1$, $2, \cdots ; j=0, \pm 1, \pm 2, \cdots)$ of $L_{2}(X, B, m)$ such that

$$
U: f_{i, j} \rightarrow f_{i, j+1} \text {. }
$$

$A$ set $B \in B$ is said to be a wandering set of positive measure if $m(B)>0$ and $T^{i} B \cap T^{i} B=\varnothing$ if $i \neq j$.

By a theorem of Hopf [3], $X$ decomposes into two disjoint $T$ invariant sets $C, D \in B$ where $C \cup D=X$, such that no $B$ subset of $C$ is a wandering set of positive measure and there exists $B \in B, B \subset D$, satisfying $\cup_{i=-\infty}^{\infty} T^{i} B=D$ and $T^{i} B \cap T^{i} B=\varnothing$ if $i \neq i$.

The sets $C, D$ also have the following property:

For every integrable $f$ with $f(x)>0$ :

$$
\sum_{i=0}^{\infty} f\left(T^{i} x\right)=\infty \text { if } x \in C \text { and } \sum_{i=0}^{\infty} f\left(T^{i} x\right)<\infty \text { if } x \in D .
$$

The sets $C, D$ (which are essentially unique) are called the conservative and dissipative parts of $X$ respectively. If $C$ is null $T$ is called completely dissipative and if $D$ is null $T$ is called completely conservative.

Let $a$ be a $\sigma$-finite sub- $\sigma$-algebra of $B$; then there is a partition $\zeta=\zeta(a)$ of $X$ into sets ( $\zeta$-fibres) such that the class of $B$ sets which are composed of whole $\zeta$-fibres coincides with $Q$. If $T^{-1} Q \subset Q$ then $T^{-1} \zeta \leqq \zeta$ (i.e., every $\zeta$-fibre is a union of $T \zeta$-fibres) [4], [5]. Let $X_{\zeta}$ be the space whose points are $\zeta$-fibres. If $T E \subset F$ where $E, F \in \zeta$ de- 
fine $T_{\zeta} C=D$, as a point map of $X_{\zeta}$. (Obviously if $x, y$ belong to the same $\zeta$-fibre then $T_{x}, T_{y}$ belong to the same $\zeta$-fibre.) Let $B_{\zeta}$ be the $\sigma$-algebra of subsets of $X_{\zeta}$ defined by $a$, and let $m_{\zeta}$ be the measure on $B_{\zeta}$ induced by $m$; then $T_{\zeta}$ is a (possibly many-one) measure preserving transformation of $\left(X_{\zeta}, B_{\zeta}, m_{\zeta}\right)$ onto itself. In fact $\left(X_{\zeta}, B_{\zeta}, m_{\zeta}, T_{\zeta}\right)$ is the homomorphic image, under a map $\phi_{\zeta}$, of $(X, \beta, m, T)$ where $\phi_{5}: x \rightarrow C$ if $x \in C$.

Let $a$ be a $\sigma$-finite sub- $\sigma$-algebra of $B$ and let $T^{-1} \propto \subset Q$ and let

$$
\begin{aligned}
& \bigvee_{i=0}^{\infty} T^{i} Q=B\left(\text { i.e., the } \sigma \text {-algebra generated by } \bigcup_{i=0}^{\infty} T^{i} Q \text { is } B\right) \\
& \bigwedge_{i=0}^{\infty} T^{-i} Q=\Re\left(\text { i.e., } \bigcap_{i=0}^{\infty} T^{-i} Q \text { is the trivial sub- } \sigma \text {-algebra of } B\right),
\end{aligned}
$$

then $T$ is called an infinite $K$-automorphism.

If (1.3) is satisfied with $Q=B$ then $T$ is called an infinite exact endomorphism and is necessarily ergodic.

If $\zeta=\zeta(a)$ and $T$ is an infinite $K$-automorphism (with respect to a) then $T_{\zeta}$ is an infinite exact endomorphism of $\left(X_{\zeta}, B_{\zeta}, m_{\zeta}\right)$. These terms extend the existing terminology for transformations of a finite measure space [2].

Let $T$ be a one-one (or many-one) measure preserving transformation of $(X, B, m)$ on to itself and let $B \in B$ be a set of positive measure such that almost all points of $B$ return infinitely often to $B$ under both positive and negative iterations of $T$, then the induced transformation $T_{B}$ defined by

$$
T_{B} x=T^{n} x \text { if } x, T^{n} x \in B \text { and } T^{i} x \in B, 0<i<n
$$

is defined for almost all points of $B$, and is a measure preserving transformation of $B-N$ onto itself for some null set $N$. Suppose further that the smallest invariant set which contains $B$ is essentially the whole space $X$, then $T$ is ergodic on $X$ if and only if $T_{B}$ is ergodic on $B-N[6]$.

If $T^{-1} a \subset a$ where $a$ is $\sigma$-finite and if $T$ is conservative, then $T_{A}$ is well defined on $A \in Q$ (where $0<m(A)<\infty$ ), $\left(T_{\zeta}\right)_{A_{\zeta}}$ is well defined on

$$
\begin{aligned}
A_{\zeta} & =\phi_{\zeta}(A) \in a_{\zeta}, \quad \text { and } \\
\left(T_{\zeta}\right)_{A_{\zeta}} & =\left(T_{A}\right)_{\zeta \cap_{A}}
\end{aligned}
$$

where $\zeta \cap A=\{E \cap A: E \in \zeta\}$ is the partition of $A$ induced by $\zeta=\zeta(Q)$. Moreover, if $\bigvee_{n=0}^{\infty} T^{n} Q=B$ then 


$$
\begin{aligned}
T_{A}^{-1}(Q \cap A) \subset Q \cap A & =\{E \cap A: E \in Q\} \text { and } \\
& \bigvee_{n=0}^{\infty} T_{A}^{n}(a \cap A)=B \cap A .
\end{aligned}
$$

\section{Ergodic analysis.}

Theorem 1. Suppose $T^{-1} Q \subset Q$ and $\bigvee_{i=0}^{\infty} T^{n} Q=Q$ where $a$ is $\sigma$ finite and suppose $A \in Q$ where $0<m(A)<\infty$ and $T_{A}$ is well defined on $A$. Suppose further that the smallest invariant set containing $A$ is the whole space $X$, then the following statements are mutually equivalent:

(i) $T$ is ergodic on $X$.

(ii) $T_{\zeta}$ is ergodic on $X_{\zeta}$.

(iii) $T_{A}$ is ergodic on $A$.

(iv) $\left(T_{A}\right)_{\zeta \cap A}=\left(T_{\zeta}\right)_{A_{\zeta}}$ is ergodic on $A_{\zeta}$.

Proof. The implications (i) $\Rightarrow$ (ii) $\Leftrightarrow$ (iv), (i) $\Leftrightarrow($ iii) $\Rightarrow$ (iv) present no difficulties and (ii) $\Rightarrow$ (i) follows from these implications and (iv) $\Rightarrow$ (iii). We show that (iv) $\Rightarrow$ (iii). Suppose $\left(T_{A}\right)_{\zeta \cap A}$ is ergodic and $T_{A}^{-1} E=E$ where $E \subset A$ and $E \in B$. Choose $\epsilon>0$ and $F \in T_{A}^{n}(a \cap A)(F \subset A)$ such that $m(F \Delta E)<\epsilon$ then $m\left(T_{A}^{-n} F \Delta E\right)<\epsilon$ where $T_{A}^{-n} F \in \mathfrak{a} \cap A$.

Consequently $E$ can be approximated arbitrarily closely by sets in $a \cap A$ and therefore $E \in Q \cap A$. Hence $E_{\zeta}$ is a $\left(T_{A}\right)_{\zeta \cap A}$ invariant set and $m_{\zeta}\left(E_{\zeta}\right)=m_{\zeta}\left(A_{\zeta}\right)$ or $m_{\zeta}\left(E_{\zeta}\right)=0$ i.e. $m(E)=m(A)$ or $m(E)=0$.

CoRollary. If $T$ is an infinite $K$-automorphism (with respect to $a$ ) then either $T$ is completely dissipative or $T$ is ergodic.

Proof. Let $D$ be the dissipative part of $X$. Let $f(x)$ be a strictly positive integrable function which is $a$ measurable. Then $D$ $=\left\{x: \sum_{i=0}^{\infty} f\left(T^{i} x\right)<\infty\right\} \in q$. Therefore $D$ is either null or essentially the whole space $X$. If $D$ is null then $T$ is completely conservative. In view of the theorem we need only show that there exists a set $A \in \mathbb{Q}$ with $0<m(A)<\infty$ such that the smallest invariant set containing $A$ is essentially the whole space. Let $E=\bigcup_{n=0}^{\infty} T^{-n} A \in Q$ then $T^{-1} E \subset E$ and since $T$ is conservative $E$ is essentially invariant. Therefore $E$ is essentially the whole space $X$.

\section{Spectral analysis.}

THEOREM 2. If $T$ is completely dissipative then $U_{T}$ has denumerable Lebesgue spectrum.

Proof. Let $\cup_{n=-\infty}^{\infty} T^{n} B=X$ where $B \in B$ and $T^{i} B \cap T^{j} B=\varnothing$ if $i \neq j$. Let $\left\{f_{n}\right\}$ be an orthonormal basis of $L_{2}(B, B \cap B, m)$ and define 


$$
\begin{aligned}
g_{n}(x) & =f_{n}(x) & & \text { if } x \in B, \\
& =0 & & \text { if } x \notin B,
\end{aligned}
$$

then $U_{T}^{m} g_{n}(x)$ vanishes outside $T^{-m} B$. Consequently $\left\{f_{n, m}\right\}=\left\{U_{T}^{m} g_{n}\right\}$ is an orthonormal basis of $L_{2}(X, B, m)$ and $U_{T} f_{n, m}=U_{T}^{m+1} f_{n}=f_{n, m+1}$.

The proofs in the remainder of this section are similar to the proofs of their 'finite' analogues, [7], [8].

Lemma 1. If $T^{-1} \propto \subset \propto$ where $Q$ is $\sigma$-finite and $T$ is ergodic then $a$ is nonatomic.

Proof. Suppose $A \in Q$ is an atom of $Q$ (i.e., if $B \in Q$ then either $B \supset A$ or $B \cap A=\varnothing)$ then $0<m(A)<\infty$ since $Q$ is $\sigma$-finite. Moreover, $T^{n} A$ is an atom of $T^{n} Q$ and since $A \in Q \subset T^{n} Q(n>0)$, either $A=T^{n} A$ or $A \cap T^{n} A=\varnothing$. If $A=T^{n} A$ for some integer $n>0$ then $X=A \cup T A$ $\cup \ldots \cup T^{n-1} A$ since the latter set is $T$ invariant. However, this would imply $m(X)<\infty$. Consequently $A \cap T^{n} A=\varnothing$ for all $n>0$ and therefore $A$ is a wandering set of positive measure contradicting the ergodicity of $T$. (The $\sigma$-algebra $B$ is nonatomic.)

LEMma 2. If $T^{-1} \propto \subset Q$ and $T^{-1} \propto \neq Q$ where $Q$ is $\sigma$-finite and $T$ is ergodic, then the orthogonal complement of $L_{2}\left(X, T^{-1} \propto, m\right)$ in $L_{2}(X, a, m)$ has infinite (denumerable) dimension.

Proof. Let $H \oplus U_{T} L=L$ where $L=L_{2}(X, a, m)$ and $U_{T} L$ $=L_{2}\left(X, T^{-1} \propto, m\right)$. Since $T^{-1} \propto \neq \emptyset$ we know that $H$ is nontrivial. Let $0 \not f \in H$ and let

$$
F=\{x: f(x) \neq 0\}
$$

so that $F \in Q$ and $m(F)>0$.

Let $\chi_{F} L=\left\{\chi_{F} g: g \in L\right\}$ and $\chi_{F} U_{T} L=\left\{\chi_{F} g: g \in U_{T} L\right\}$, then since $F$ contains no atoms of $Q, \chi_{F} L$ has infinite dimension.

Define $H_{1} \subset H$ by

$$
H_{1} \oplus \chi_{F} U_{T} L=\chi_{F} L
$$

If $\chi_{F} U_{T} L$ has finite dimension then $H_{1}$ has infinite dimension. Suppose $\chi_{F} U_{T} L$ has infinite dimension and choose an independent basis $\left\{\chi_{F} f_{n}\right\} \subset \chi_{F} U_{T} L\left(f_{n} \in U_{T} L\right)$ such that the functions $f_{n}$ are bounded then $\left\{f f_{n}\right\}$ is an independent set and each function $f f_{n}$ is $Q$ measurable, square integrable and vanishes outside $F$. Moreover, for each $g \in U_{T} L$

$$
\int f f_{n}\left[\chi_{F} g\right]-d m=\int f\left[\bar{f}_{n} g\right]-d m=0
$$


since $f \in H$ and $\bar{f}_{n} g \in U_{T} L$. Therefore $\left\{f f_{n}\right\}$ is an independent sequence of functions in $H_{1} \subset H$ i.e., $H$ has infinite dimension.

THEOREM 3. If $T$ is an infinite $K$-automorphism then $U_{T}$ has denumerable Lebesgue spectrum.

Proof. By the Corollary to Theorem 1, $T$ is either ergodic or completely dissipative. In the latter case $U_{T}$ has denumerable Lebesgue spectrum by Theorem 2 . In the former case we can invoke Lemmas 1 and 2. Denote by $H$, the orthogonal complement of $U_{T} L$ in $L$ where $L=L_{2}(X, a, m)$. Then $H$ has infinite denumerable dimension. Let $\left\{f_{n}\right\}$ be an orthonormal basis of $H$. It is not difficult to see that $\bigcap_{n=0}^{\infty} U_{T}^{n} L$ is the trivial subspace of $L$ and $U_{n=0}^{\infty} U_{T}^{-n} L$ is dense in $L_{2}(X, \Theta, m)$. Repeated application of the decomposition $L=U_{T} L \oplus H$ yields

$$
L(X, \beta, m)=\bigoplus_{n=-\infty}^{\infty} U_{T}^{n} H
$$

and therefore $\left\{f_{n, m}\right\}=\left\{U_{T}^{m} f_{n}\right\}$ is an orthonormal basis such that $U_{T} f_{n, m}=U_{T}^{m+1} f_{n}=f_{n, m+1}$.

4. Conclusion. It only remains to give examples of ergodic infinite $K$-automorphisms, and these are provided by the class of irreducible aperiodic recurrent Markov chains, with stationary transition probabilities and countable state space, which preserve a $\sigma$-finite infinite measure [9].

If $T$ is an infinite $K$-automorphism [infinite exact endomorphism] then $T^{(k)}=T \times T \times \cdots \times T$ ( $k$ times) is an infinite $K$-automorphism [infinite exact endomorphism]. If $T$ is an infinite $K$-automorphism then for some integer $k$ it may happen that $T^{(k)}$ is ergodic but $T^{(k+1)}$ is not ergodic. In fact this happens when $T^{(k)}$ is conservative and $T^{(k+1)}$ is not conservative. This is precisely the phenomenon analysed for Markov chains in [10]. It follows that the ergodic index [10] is not a spectral invariant.

\section{REFERENCES}

1. P. R. Halmos, Problems, Proceedings of a Symposium on Ergodic Theory, Academic Press, New York, 1963.

2. V. A. Rohlin, New progress in the theory of transformations with invariant measure, Uspehi Mat. Nauk. 15 (1960), 1-26; Russian Math. Surveys. 15 (1960), 1-22.

3. E. Hopf, Ergodentheorie, Ergebnisse der Mathematik, Springer, Berlin, 1937.

4. D. Blackwell, On a class of probability spaces, Proc. 3rd Berkeley Sympos., Vol. 2, 1956, pp. 1-6.

5. V. A. Rohlin, On the fundamental ideas of measure theory, Mat. Sb. (N.S.) 25 (1949), 107-150; Amer. Math. Soc. Transl. (1952), no. 71. 
6. S. Kakutani, Induced measure preserving transformations, Proc. Imp. Acad. Sci. Tokyo 19 (1943), 635-641.

7. K. Jacobs, Lecture notes on ergodic theory (mimeographed), Mathematisk Institut, Aarhus Universitet, Aarhus, Denmark, 1963.

8. V. A. Rohlin, Exact endomorphisms of a Lebesgue space, Izv. Akad. Nauk. SSSR. 25 (1961), 499-530. (Russian.)

9. D. Blackwell and D. Freeman, The tail $\sigma$-field of a Markov chain and a theorem of Orey, Ann. Math. Statist. 35 (1964), 1291-1295.

10. S. Kakutani and W. Parry, Infinite measure preserving transformations with "mixing," Bull. Amer. Math. Soc. 69. (1963), 752-756.

The University, Birmingham, England 